\title{
The Solar Orbiter mission and its prospects for helioseismology
}

\author{
J. Woch ${ }^{1, \star}$ and L. Gizon ${ }^{1}$ \\ Max-Planck-Institut für Sonnensystemforschung, Max-Planck-Str. 2, D-37191 Katlenburg-Lindau, Germany
}

Received 22 Dec 2006, accepted 2 Jan 2007

Published online later

Key words Solar Orbiter - Helioseismology

Solar Orbiter is intended to become ESA's next solar mission in heritage of the successful SOHO project. The scientific objectives of the mission, its design, and its scientific payload are reviewed. Specific emphasis is given to the perspectives of Solar Orbiter with respect to helioseismology.

(c) 2007 WILEY-VCH Verlag GmbH \& Co. KGaA, Weinheim

\section{Introduction}

The successful ESA/NASA solar observatory SOHO which was launched in 1995 has provided and still is providing an unprecedented breadth and depth of information about the Sun, from the Sun's interior, through the hot and dynamic atmosphere, to the solar wind and its interaction with the interstellar medium. The SOHO mission still constitutes the prime focus for the research of the European solar science community. Specifically, data returned from the MDI instrument onboard SOHO have given a tremendous push to the field of helioseismology. The Solar Orbiter mission has been configured as the logical advancing step in the exploration of the Sun after SOHO. By approaching the Sun as close as 48 solar radii ( $0.22 \mathrm{AU})$, Solar Orbiter will view the solar atmosphere with a spatial resolution of less than $150 \mathrm{~km}(1 \mathrm{arcsec})$ and will perform the closest ever in-situ measurements. In the course of the mission the inclination of the spacecraft orbit to the ecliptic will incrementally increase to reach heliographic latitudes larger than $30^{\circ}$ which will provide the first ever views of the Sun's polar regions. A further unique aspect of the mission is the combination of comprehensive remote sensing and in-situ instrumentations allowing to trace solar features from the photosphere to interplanetary space.

Of high interest for helioseismology is the large range of spacecraft-Sun-Earth angles covered by Solar Orbiter. In combination with observation from Earth (ground-based or near-Earth orbit), Solar Orbiter will offer the opportunity for stereoscopic helioseismology.

\section{Scientific objectives}

Missions such as Helios, Ulysses, Yohkoh, SOHO, TRACE and RHESSI have advanced significantly our understanding of the various atmospheric layers of the Sun, the solar wind

\footnotetext{
^ Corresponding author: e-mail: woch@mps.mpg.de
}

and the three-dimensional heliosphere. Further progress is to be expected from missions like STEREO, Solar-B, and the Solar Dynamics Observatory (SDO), the first of NASAs Living With a Star (LWS) missions.

Each of these missions has a specific focus, each fulfilling its part within a coordinated solar and heliospheric research effort. Solar Orbiter with its unique orbit and advanced suit of in-situ and remote sensing instruments constitute an important augmentation to this effort. Solar Orbiter will for the first time:

- Explore the uncharted innermost regions of our solar system

- Study the Sun from close-up

- Fly by the Sun tuned to its rotation and examine the solar surface and the space above from a corotating vantage point - Provide images and spectral observations of the Suns polar regions from out of the ecliptic.

According to the ESA Solar Orbiter Science Requirement Document the top-level scientific goals of the Solar Orbiter mission are to:

- Determine the properties, dynamics and interactions of plasma, fields and particles in the near-Sun heliosphere

- Investigate the links between the solar surface, corona and inner heliosphere

- Explore, at all latitudes, the energetics, dynamics and finescale structure of the Sun's magnetized atmosphere - Probe the solar dynamo by observing the Sun's high-latitude field, flows and seismic waves.

\section{Mission design}

Solar Orbiter is a three-axis stabilized spacecraft. On the sun-facing side a heat shield protects the spacecraft and its payload from the compared to Earth 25 times higher radiation. The heat shield has to provide the apertures for the remote sensing instruments.

According to the current baseline (as at December 2006) Solar Orbiter is scheduled to be launched in May 2015 on 


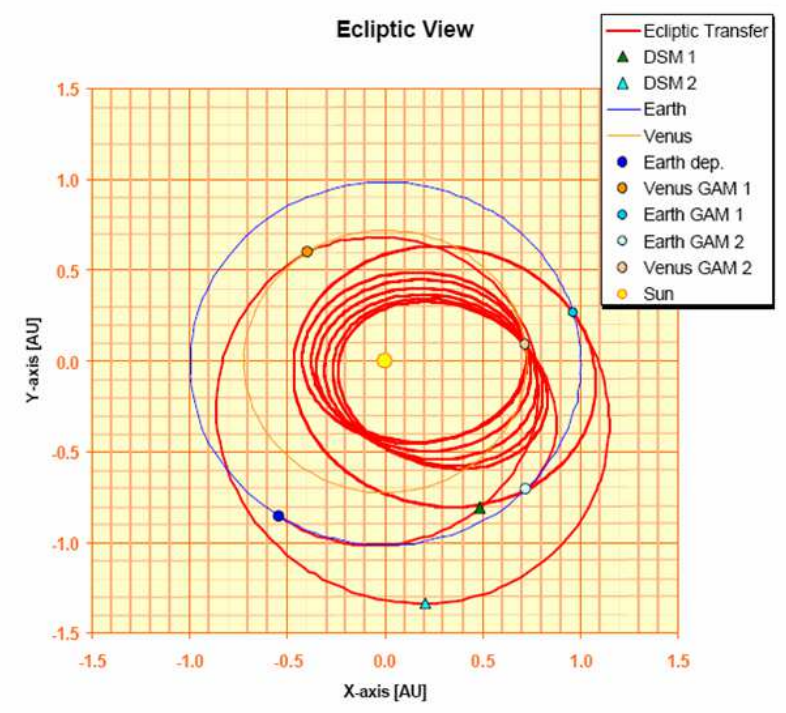

Fig. 1 The projection of the orbit of Solar Orbiter into the ecliptic plane. Earth and Venus orbit as well as the position of various spacecraft manoeuvres are indicated. (Figure adapted from a presentation by R. Marsden.)

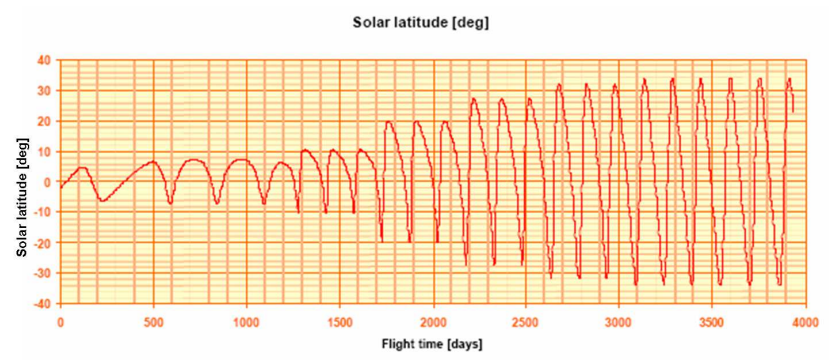

Fig. 2 The solar latitude of Solar Orbiter as a function of mission duration. (Figure adapted from a presentation by R. Marsden.)

a Soyuz-Fregat 2-1b. The spacecraft will use a chemical propulsion system and multiple gravity assist manoeuvres at Venus and Earth to reach its science orbit. The initial cruise to the science orbit will last 3.4 years. In the science phase, Solar Orbiter will be in a 3:2 resonant orbit with Venus (period 149.8 days). The Venus fly-bys are used to slowly increase the inclination of the orbit. The total mission duration will be approximately 10 years. The minimum perihelion distance during the science phase will be 48 solar radii $(0.22 \mathrm{AU})$ with a maximum solar latitude of $34^{\circ}$. The projection of the baselined orbit into the ecliptic $x-y$ plane and the solar latitude as a function of mission duration are shown in Figure 1 and Figure 2, respectively.

Figure 3 shows the time profile of the maximum solar latitude reached in each orbit together with a predicted profile of the solar activity based on the sunspot number. In 2015, Solar Orbiter will be launched into the solar activity minimum, with the cruise phase extending through the minimum phase. Solar activity is expected to increase again at the start of the science phase. While the activity further de- velops, Solar Orbiter will advance to higher latitudes. During the declining phase of the activity cycle the spacecraft will reach highest latitudes. This offers the possibility to study the transition from an active to a quiet Sun from a high-latitude vantage point. The current mission baseline allows observations to be carried out for about 10 days around the perihelion passes and/or the high-latitude segments of each of the science orbits.

\section{Scientific payload}

The current Solar Orbiter scientific payload ia a comprehensive set of remote sensing and in-situ instrumentation. It offers the unprecedented opportunity for complementary observation of solar processes as they evolve from the photosphere into near-Sun interplanetary space. The near-Sun interplanetary measurements together with simultaneous remote sensing observations of the Sun will permit to disentangle spatial and temporal variations during the corotation phases. They will allow to understand the characteristics of the solar wind and energetic particles and link them to the plasma, neutral atmosphere and magnetic conditions in their source regions on the Sun. The Solar Orbiter remote sensing instruments will deliver images of the solar atmosphere with a spatial resolution of 0.5 arcsec pixels, equivalent to $80 \mathrm{~km}$ on the Sun at $0.222 \mathrm{AU}$, which means resolving solar features as small as $160 \mathrm{~km}$ (ESA Solar Orbiter Science Requirements Document). The reference core payload, their scientific goals and allocated resources (according to ESA Solar Orbiter Payload Definition Document) are shown in Figure 4.

\section{Current status}

Solar Orbiter is currently targeted for a launch in 2015. In July 2006 ESA has issued a letter of intent (LOI) to propose instruments for the Solar Orbiter mission with deadline for responses in September 2006. ESA has received 23 LOIs, documenting the interest of the solar community in Solar Orbiter. However, due to the financial constrains of ESA, the mission is currently reviewed again and undergoes a consolidation phase with the aim of cost reduction. Beside descoping, one potential way of limit costs to ESA is to merge Solar Orbiter with the Sentinel mission of NASA. The consolidation phase will be used to advance the readiness of the mission, specifically in respect to the critical heat shield - instrument interfaces by further refinement of the instruments based on interactions with potential principal investigator teams. Final decision on Solar Orbiter is expected in November 2007. 
Ballistic Mission

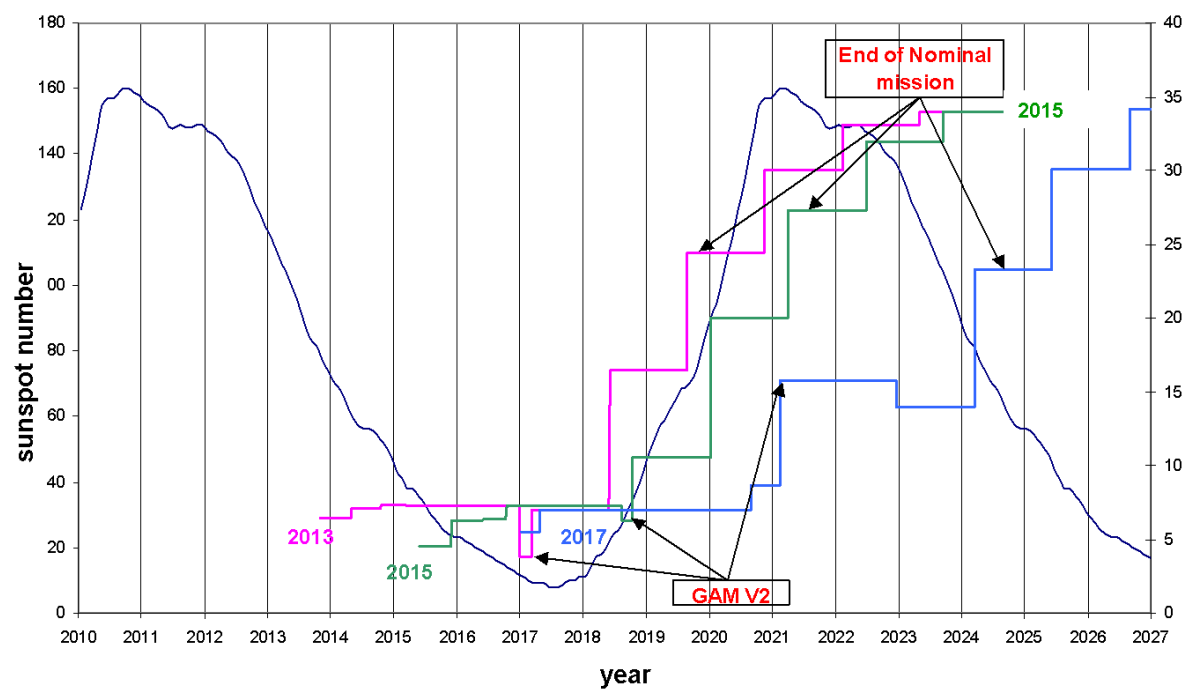

Fig. 3 The maximum solar latitude reached for each of the science orbits as a function of mission duration for various launch dates together with the predicted sunspot number. (Figure adapted from a presentation by R. Marsden.)

\section{The VIM instrument and its perspective for helioseismology}

The Visible Light Imager and Magnetograph (VIM) is one of the remote sensing instruments in the core reference payload of Solar Orbiter. VIM will measure the structure, dynamics and evolution of the full magnetic vector and of the flow field in the solar photosphere as well as waves and oscillations that penetrate into the solar interior. VIM will give the first view of the magnetic and velocity field in the polar regions. VIM consists of two telescopes, a High Resolution Telescope (HRT) and a Full Disk Telescope (FDT), both of which feed a filtergraph. They provide high resolution measurements within a limited field-of-view and low resolution measurements covering the full solar disk. Maps of the the full photospheric magnetic field vector, the line-of-sight velocity, and the continuum intensity will be obtained with a cadence of less than a minute. This requires recording linearly and circularly polarized intensities (Stokes vectors) at different wavelengths in a Zeeman-sensitive photospheric spectral line and the nearby continuum.

Among the Solar Orbiter instrumentation, VIM will deliver the most relevant data for helioseismology.

The method of global-mode helioseismology (Christensen-Dalsgaard 2002) has allowed to map the solar differential rotation as a function of latitude and radial distance throughout most of the convection zone with a very good level of precision, while the sound speed profile averaged over spheres has been measured down to the solar core. At high heliographic latitudes, however, the picture of the solar interior is far from complete and is fuzzier with greater depth. In particular, global mode frequency inversions for rotation cease to be accurate above $70^{\circ}$ latitude and are uncertain in the radiative interior.

The developing science of local helioseismology (Gizon $\&$ Birch 2005), which aims at producing three-dimensional images of the solar interior, has provided information in the upper third of the convective envelope and only at latitudes less than $50^{\circ}$. High latitudes are difficult to study because the sensitivity of the line-of-sight Doppler measurements to the nearly radial pulsations drops as a function of centerto-limb distance. In addition, the reduction of the effective spatial sampling on the Sun toward the solar limb implies that moderate to high degree modes are more difficult to detect away from disk center in the center-to-limb direction. That measurements are difficult to make close to the solar limb has implications not only for studies at high latitudes, but also for probing deep into the interior. Indeed, acoustic ray bundles that penetrate deep inside the Sun connect surface locations that are separated by large horizontal distances: less signal at high latitudes means a reduced ability to probe deep at moderate latitudes. In short, much remains to be learned above $50^{\circ}$ heliographic latitude and for radial distances less than, say, 0.9 solar radius.

The VIM instrument will return the necessary Doppler images of the polar regions of the Sun. In addition, VIM Doppler images combined with Doppler images taken from Earth will allow to demonstrate the concept of stereoscopic helioseismology. Thus, VIM data as input for helioseimic methods offer the unique opportunity to address some outstanding key questions of solar physics:

(1) Internal rotation at high latitudes: The large-scale differential rotation is an essential dynamical property of the Sun. Inversions of global-mode frequency splittings support the idea that rotation is special at heliographic latitudes 


\begin{tabular}{|c|c|c|c|c|c|c|}
\hline Instrument & Acronym & Science goals & $\begin{array}{l}\text { Spectral band - } \\
\text { Particle range }\end{array}$ & $\begin{array}{c}\text { Total } \\
\text { Mass } \\
\text { [kg] }\end{array}$ & $\begin{array}{l}\text { Power } \\
\text { (2) }[W]\end{array}$ & $\begin{array}{l}\text { TM (3) } \\
\text { [kbps] }\end{array}$ \\
\hline \multicolumn{7}{|l|}{ In-Situ Instruments } \\
\hline Solar Wind Plasma Analyzer & SWA & $\begin{array}{l}\text { Investigation of kinetic properties and } \\
\text { composition (mass and charge states) of solar } \\
\text { wind plasma }\end{array}$ & $\begin{array}{l}\mathrm{e}-: 0.001-5 \mathrm{keV} / \mathrm{q} \\
\mathrm{p}+, \alpha: 0.2-20 \mathrm{keV} / \mathrm{q} \\
\text { ions: } 0.5-100 \mathrm{keV} / \mathrm{q}\end{array}$ & 16.5 & 15.5 & 7 \\
\hline $\begin{array}{l}\text { Radio and Plasma Wave } \\
\text { Analyzer }\end{array}$ & RPW & $\begin{array}{l}\text { Investigation of radio and plasma waves } \\
\text { including coronal and interplanetary } \\
\text { emissions }\end{array}$ & $1 \mathrm{~Hz}$ to $10 \mathrm{MHz}$ & 13.0 & 7.0 & 5 \\
\hline Magnetometer & MAG & Investigation of the solar wind magnetic field & $\begin{array}{l}\text { Time resolution: } 16 \\
\text { samples / sec normal ops } \\
\text { Absolute precision: } 1 \mathrm{nT}\end{array}$ & 2.1 & 1.5 & 0.8 \\
\hline Energetic Particle Detector & EPD & $\begin{array}{l}\text { Investigation of the origin, acceleration and } \\
\text { propagation of solar energetic particles }\end{array}$ & $\begin{array}{l}0.002-100 \mathrm{MeV} / \text { nucleon } \\
\text { in } 5 \text { units }(\mathrm{e}-/+, \mathrm{p}+\text {, ions) }\end{array}$ & 15.1 & 14.5 & 3.1 \\
\hline Dust Particle Detector & DPD & $\begin{array}{l}\text { Investigation of the flux, mass and major } \\
\text { elemental composition of near-Sun dust }\end{array}$ & $10-15-10-6$ gr & 1.8 & 6 & 0.1 \\
\hline $\begin{array}{l}\text { Neutron Gamma ray } \\
\text { Detector }\end{array}$ & NGD & $\begin{array}{l}\text { Investigation of the characteristics of low- } \\
\text { energy solar neutrons, and solar flare } \\
\text { processes }\end{array}$ & $\begin{array}{l}\text { n: } 0.6-20 \mathrm{MeV} \\
\text { Gamma: } 0.05-10 \mathrm{MeV}\end{array}$ & 5.5 & 5.5 & 0.4 \\
\hline \multicolumn{7}{|l|}{ Remote-sensing Instruments } \\
\hline $\begin{array}{l}\text { Visible Imager \& } \\
\text { Magnetograph }\end{array}$ & VIM & $\begin{array}{l}\text { Investigation of the magnetic and velocity } \\
\text { fields in the photosphere }\end{array}$ & $\begin{array}{l}400-700 \mathrm{~nm}(1 \mathrm{narrow} \\
\text { pass-band of } 5-10 \mathrm{~nm})\end{array}$ & 30.4 & 35 & 20 \\
\hline EUV Spectrometer & EUS & $\begin{array}{l}\text { Investigation of properties of the solar } \\
\text { atmosphere }\end{array}$ & $\begin{array}{l}17-100 \mathrm{~nm}(2-3 \text { narrow } \\
\text { bands) }\end{array}$ & 18.0 & 35 & 17 \\
\hline EUV Imager & EUI & $\begin{array}{l}\text { Investigation of the solar atmosphere using } \\
\text { high resolution imaging in the EUV }\end{array}$ & $\begin{array}{l}13.3 \mathrm{~nm}, 17.4 \mathrm{~nm}, 30.4 \\
\mathrm{~nm}(3 \text { bands) }\end{array}$ & 20.4 & 28 & 20 \\
\hline Visible Coronograph & $\mathrm{COR}$ & $\begin{array}{l}\text { Investigation of coronal structures using } \\
\text { polarized brightness measurements in Vis }\end{array}$ & $\begin{array}{l}450-600 \mathrm{~nm}+121.6 \mathrm{~nm} \\
\text { and } 30.4 \mathrm{~nm} \text { (optional) }\end{array}$ & 18.3 & 30 & 10 \\
\hline $\begin{array}{l}\text { Spectrometer Telescope } \\
\text { Imaging X-ray }\end{array}$ & STIX & $\begin{array}{l}\text { Investigation of energetic electrons near the } \\
\text { Sun, and solar x-ray emission }\end{array}$ & $3-150 \mathrm{keV}$ & 4.4 & 4 & 0.2 \\
\hline Payload Support Elements & PSE & -- & - & 26.0 & 4 & - \\
\hline TOTAL & & & & 171.5 & 186.0 & 83.6 \\
\hline
\end{tabular}

Fig. 4 The scientific core reference payload of Solar Orbiter (adapted from the ESA Solar Orbiter Payload Definition Document).

above $70^{\circ}$. In particular, some inversions suggest the existence of a buried jet-like zonal flow (Schou 1999) and a surprisingly slow rotation rate above $75^{\circ}$ compared to a smooth extrapolation from lower latitudes (Schou et al. 1998; Birch \& Kosovichev 1998). In addition, the near-surface radial gradient of the angular velocity, may switch sign near latitude $50^{\circ}$ (Corbard \& Thompson 2002). These results have been derived using global-mode techniques, which do not necessarily require local observations at high latitudes. Using observations out of the ecliptic plane together with techniques of local helioseismology, VIM may offer the opportunity to check the validity of the global-mode frequency inversions at high latitudes. Of course, direct Doppler measurements will also give important information about the surface rotation rate.

(2) Meridional circulation: Surface Doppler measurements have shown the existence of a meridional flow from the equator to the poles with a maximum amplitude of about
$15 \mathrm{~m} / \mathrm{s}$ near $30^{\circ}$ latitude. Meridional circulation plays a role in the evolution of the large-scale surface magnetic field. In flux-transport dynamo models based on the BabcockLeighton mechanism, it carries the poloidal field generated at the surface down to the bottom of the convection zone, transports the toroidal magnetic field equatorward, and sets the period of the solar cycle (Dikpati \& Charbonneau 1999). Due to mass conservation, the meridional flow amplitude at the base of the convection zone is expected to be extremely small. Techniques of local helioseismology are sensitive to the first order effect of meridional circulation. However, the meridional flow at latitudes above $50^{\circ}$ is poorly known and should be an important target for VIM. One question is whether the meridional flow has a single-cell or multi-cell structure. The determination of the latitudinal component of the meridional flow in the near polar regions is likely to be extremely difficult (a very small signal is expected). 
(3) Polar region variability: The polar regions appear to be very dynamical. There is evidence that the differential rotation near the poles may vary on a short time scale (Ye \& Livingston 1998). The magnetic properties of the polar regions at solar minimum determine the strength of the forthcoming solar maximum (Schatten 1998); a new sunspot cycle starts in the polar regions. Thus, studying the dynamics and variability of the polar regions will help us understand how the solar dynamo works. Using global-mode helioseismology, it was found that there may be a significant variation of the subsurface high-latitude rotation as a function of the phase of the solar cycle (Birch \& Kosovichev 1998; Schou 2003). Does the meridional circulation, which is known to vary with time near active latitudes, also vary at high latitudes? In particular, does it exhibit multi-cell structures that come and go with the phase of the solar cycle? We may also ask about flow patterns across the poles. Answering these questions will require long-term monitoring of the polar regions.

(4) Convection at high latitudes: Near-surface convective flows act as an important driver of solar activity. By controlling the evolution of the magnetic network, supergranulation affects chromospheric and coronal activity. Nearsurface convective flows could support local dynamo action too. Is the dynamics of supergranulation different in the polar regions where the effect of the Coriolis force is expected to be much stronger than that at lower latitudes? What is the dynamical coupling between near-surface flows in the near polar regions and photospheric/coronal magnetism? Flows at supergranular scales can be measured with seismic holography or time-distance helioseismology with sufficient temporal resolution. However, a good spatial resolution does require that f-modes be observed (Duvall \& Gizon 2000). Possibly, flows at larger spatial scales than supergranulation can be measured with the technique of ring diagram analysis.

(5) Structure and variability of the Tachocline, dynamo flows: The solar tachocline is believed to be the seat of the generation of the global magnetic field (Gilman 2005) and is thus an important target for helioseismological studies. A proper determination of the figure of the solar tachocline obviously requires high latitude information. It has recently been suggested that prograde fluid jets may be generated in the solar tachocline at high latitudes, which may be measurable by methods of helioseismology (Rempel \& Dikpati 2003; Christensen-Daalsgard et al. 2004). The tachocline also appears to support quasi-periodic oscillations with a period of approximately 1.3 year (Howe et al. 2000), which may be a deep extension of the torsional oscillations. There are indications that surface magnetic fields often cluster at a few specific longitudes for several rotation periods (Henney \& Harvey 2002). This may indicate the presence of an underlying non-axisymmetric magnetic structure and could be a manifestation of non-axisymmetric modes of the dynamo (Ruzmaikin 1998; Berdyugina et al. 2006). Local helioseismology is the appropriate tool to search for longitudi- nal variations deep in the convection zone. Stereoscopic local helioseismology may be an important advance to detect the signature of deep dynamo flows and their longitudinal variations inside the Sun.

(6) Stereoscopic helioseismology: Stereoscopic helioseismology would benefit both global and local methods of helioseismology. Stereoscopy requires several observatories. Both SDO-HMI and GONG (or another ground-based facility) are expected to be operational at the time of Solar Orbiter operations. Combining Solar Orbiter with any one of them will open new windows into the Sun. Looking at the Sun from two distinct viewing angles results in an increase of the observed fraction of the Sun's surface. Global helioseismology would naturally benefit from this situation, since the modes of oscillation would be easier to disentangle (reduction of spatial leaks). The precision on the determination of the mode frequencies would improve at high frequencies for all spherical harmonic degrees. Spatial masks for extracting acoustic modes will be closer to optimal. With stereoscopic local helioseismology, new acoustic ray paths can be considered to probe deeper layers in the interior. Observations from two widely different viewing angles allow the probing of the solar interior at any depth, and in particular the solar tachocline (see above). It even becomes possible to target regions in the solar core. This aspect of seismic stereoscopy is revolutionary. The other aspect of stereoscopy is that it is possible to observe a common area from two different viewing angles. This case is useful to understand systematic errors and line-of-sight projection effects in local helioseismology. It is also potentially important to minimize the contribution of convection noise in spectra of solar oscillations. Finally, there is the possibility of observing the same area with two different spectral lines formed at two heights in the solar atmosphere to study vertical wave propagation. In essence, VIM observations in combination with other solar Doppler imaging have the capability to demonstrate the promising concept of stereoscopic helioseismology.

\section{Requirements for helioseismology}

Many of the above scientific objectives are difficult to accomplish. Which objective can be fulfilled and to which extent depends on the actual performance of the VIM instrument. High quality image stabilization, accurate knowledge of pointing and precise timing are a prerequisite for useful measurements.

The observation duration is one of the most critical parameter: the larger the observation interval $T$, the smaller the contribution of random noise to helioseismic measurements. For nearly all quantities of interest, the level of random noise due to the stochastic nature of solar oscillations behaves like $T^{-1 / 2}$. The current mission baseline of Solar Orbiter allows observations to be carried out for about $T=10$ days during each orbit, near perihelion. Studies of the solar nearsurface layers require days to weeks of data. 
Investigations of the deep interior typically require durations from weeks to months. In practice, the longer the data coverage, the better. It would be very meaningful to at least consider the possibility of making observations during the cruise phase and outside perihelion passages, even at a very low telemetry rate. Line-of-sight vectors other than EarthSun are prefered for stereoscopic helioseismology.

The temporal sampling needs to be at least as short as 1 minute to make possible observations of all solar waves with significant power; faster sampling with, say, $40 \mathrm{~s}$ may be desirable to detect the highest frequency waves. The use of high-frequency waves is potentially important to study magnetic effects and to provide more localized information.

The field of view must be large to resolve the low-degree modes that penetrate deep into the interior, as well as to ensure the study of large scale solar inhomogeneities. A large field of view also gives the ability to track local areas on the Sun over long periods of time to compensate for the spacecraft motion in frames that co-rotate with the Sun. A fulldisk view is ideal, especially to make the most of stereoscopic analyses.

The spatial resolution measured on the Sun's surface is a function of ccd pixel size and center-to-limb distance. Solar oscillations have significant power at all wavelengths above, say, $5 \mathrm{Mm}$ (f-mode frequency at $3 \mathrm{mHz}$ ). Typically, a spatial sampling of $1 \mathrm{Mm}$ (measured on the Sun) is sufficient for nearly all applications of helioseismology. A higher spatial sampling is not needed, while a lower one is still acceptable for a large range of applications that involve longwavelength deep-penetrating modes of oscillation.

The telemetry rate will likewise have critical impact. The limited telemetry rates require powerful lossless and lossy data compression techniques. Intelligent methods of spatial binning, Fourier filtering and low-pass filtering have to be applied to match the data volume to the telemetry rates.

\section{References}

Berdyugina, S.V., Moss, D., Sokoloff, D., Usoskin, I.G.: 2006, Astron. Astrophys. 445, 703

Birch, A.C., Kosovichev, A.G.: 1998, Astrophys. J. 503, L187

Christensen-Dalsgaard, J.: 2002, Rev. Mod. Phys. 74, 1073

Christensen-Dalsgaard, J., Corbard, T., Dikpati, M., Gilman, P. A., Thompson, M.: 2004, in Proc. SOHO 14/GONG 2004, ESA SP-559, 376

Corbard, T., Thompson, M.J.: 2002, Solar Physics 205, 211

Dikpati, M., Charbonneau, P.: 1999, Astrophys. J. 518, 508

Duvall, T.L., Gizon, L.: 2000, Solar Phys. 192, 177

Gilman, P.A.: 2005, Astron. Nachr. 326, 208

Gizon, L., Birch, A.C.: 2005, Living Rev. Solar. Phys. 2, 6

Henney, C.J., Harvey, J.W.: 2002, Solar Phys. 207, 199

Howe, R., Christensen-Dalsgaard, J., Hill, F., et al.: 2000, Science 287, 2456

Rempel, M., Dikpati, M.: 2003, Astrophys. J. 584, 524

Ruzmaikin, A.: 1998, Solar Phys. 181, 1
Schatten, K.: 1998, ASP Conf. Ser. 154, The Tenth Cambridge Workshop on Cool Stars, Stellar Systems and Sun-like Stars, R. Donahue and J. Bookbinder (eds.), 1315

Schou, J.: 1999, Astrophys. J. 523, L181

Schou, J.: 2003, in Stellar astrophysical fluid dynamics, ed. M. J. Thompson, J. Christensen-Dalsgaard (CUP, Cambridge, UK), 247

Schou, J., Antia, H.M., Basu, S., et al.: 1998, Astrophys. J. 505, 390

Ye, B., Livingston, W.: 1998, Solar Phys. 179, 1 